\title{
PERFIL SOCIODEMOGRÁFICO E CLÍNICO-EPIDEMIOLÓGICO DA POPULAÇÃO EM SITUAÇÃO DE RUA COM TUBERCULOSE
}

\section{Gleiciene Oliveira Borges ${ }^{1}$; Victória Lima Mendes Leite²; Lucas Souza da Silva ${ }^{3}$; Priscila Rodrigues Tavares ${ }^{4}$; Ana Carolina da Silva Souza ${ }^{5}$; Ana Júlia Góes Maués ${ }^{6}$, Izabela Moreira Pinto $^{7}$; Solino Ansberto Coutinho Junior ${ }^{8}$ Marcos José Risuenho Brito Silva ${ }^{9}$; Marcelo Williams Oliveira de Souza ${ }^{10}$.}

${ }^{1}$ Acadêmica de Enfermagem. Universidade do Estado do Pará - UEPA. Belém, Pará.

${ }^{2}$ Acadêmica de Enfermagem. Universidade do Estado do Pará - UEPA. Belém, Pará.

${ }^{3}$ Acadêmico de Enfermagem. UNIFAMAZ. Belém, Pará.

${ }^{4}$ Acadêmica de Enfermagem. Universidade do Estado do Pará - UEPA. Belém, Pará.

${ }^{5}$ Acadêmica de Enfermagem. Universidade Federal do Pará - UFPA. Belém, Pará.

${ }^{6}$ Acadêmica de Enfermagem. Universidade do Estado do Pará - UEPA. Belém, Pará.

${ }^{7}$ Acadêmica de Enfermagem. Universidade do Estado do Pará - UEPA. Belém, Pará.

${ }^{8}$ Acadêmico de Enfermagem. Faculdade Cosmopolita. Belém, Pará.

${ }^{9}$ Enfermeiro. Universidade do Estado do Pará - UEPA. Belém, Pará.

${ }^{10}$ Enfermeiro. Universidade Federal do Pará - UFPA. Belém, Pará.

DOI: 10.47094/IICNNESP.2021/134

\section{RESUMO}

A População em situação de rua é socialmente vulnerável, o que potencializa risco de contágio da tuberculose, caracterizada como problema de saúde pública mundial. O trabalho visa analisar o perfil sociodemográfico e clínico-epidemiológico da população em situação de rua com tuberculose no período de 2017 a 2019. Trata-se de um estudo descritivo, transversal, com abordagem quantitativa, com uso de dados secundários sobre a população de rua e a população geral, obtidos no banco de dados da plataforma Tabnet/Datasus trabalhados por meio de estatística descritiva. De 2017 a 2019 foram notificados 14.546 casos de tuberculose no estado, sendo 190 pessoas em situação de rua. Nesta população foram elevadas as taxas de abandono, uso de drogas ilícitas e coinfecção TB/HIV; enquanto o percentual de cura foi menor. A vulnerabilidade social expõe pessoas ao adoecimento e efeitos negativos da tuberculose.

PALAVRAS-CHAVE: Perfil de Saúde. População em situação de rua. Tuberculose.

ÁREA TEMÁTICA: Epidemiologia 


\section{INTRODUÇÃO}

A Política Nacional para a População em Situação de Rua (PNPSR) define esse grupo populacional como pessoas que tem em comum a pobreza extrema e o uso de logradouros públicos e áreas degradadas como espaço de moradia e de sustento, de forma temporária ou permanente. A incidência da População em Situação de Rua (PSR) no Brasil ocorre por fatores econômicos, dependência química, conflitos familiares, entre outros. Este fenômeno ocorre principalmente nos grandes centros urbanos do país.

As limitações, sofrimentos físicos e emocionais, potencializam o risco de contágio de doenças, que se manifestam de forma mais precoce e severa na PSR. Dentre essas doenças, destacase a tuberculose, uma doença infectocontagiosa causada pelo Mycobacterium tuberculosis ou Bacilo de Koch, sua transmissão ocorre por vias aéreas e o órgão mais afetado é o pulmão. A TB é um problema de saúde pública mundial e acomete milhares de pessoas que ainda morrem em decorrência da doença.

No Brasil, em 2019 foram diagnosticados 73.864 casos novos de TB, com coeficiente de incidência de 35 casos/100 mil habitantes. O estado do Pará está entre os estados com maior coeficiente de incidência da doença, o qual está acima de 51 casos/100 habitantes. Em 2019, no Pará, foram registrados 4459 casos novos de TB e cerca de 213 óbitos. A obtenção de dados epidemiológicos, permite a identificação de particularidades de cada território, a qual é fundamental para o planejamento e implantação de estratégias e ações sobre tuberculose na PSR (BRASIL, 2019).

Desse modo, o presente trabalho tem como objetivo analisar o perfil sociodemográfico e clínico-epidemiológico da população em situação de rua com tuberculose no Estado do Pará, no período de 2017 a 2019.

\section{METODOLOGIA}

Trata-se de um estudo descritivo, transversal, com abordagem quantitativa, com uso de dados secundários do Sistema de Informação de Agravos de Notificação (SINAN) que permitem identificar a realidade epidemiológica de determinada área geográfica disponibilizados na plataforma Tabnet/ Datasus que atua como um tabulador genérico de domínio público possibilitando a organização de informações.

Nesse contexto, definiu-se o objeto de estudo dados obtidos sobre a situação da tuberculose na PSR no período de 2017-2019, formando o banco de dados a partir da tabulação de informações epidemiológicas, morbidade e os casos de tuberculose desde 2001, posteriormente, obteve-se amostras sobre tuberculose na PSR e na população geral (PG).

O primeiro perfil investigado foi o sociodemográfico, composto pelas variáveis: sexo, raça, faixa etária e escolaridade. O presente estudo utilizou informações fornecidas em bases secundárias, portanto não envolveu pesquisa direta com pessoas e/ou animais, obedecendo assim os princípios éticos da Resolução do Conselho Nacional de Saúde (CNS) nº 510, de 7 de abril de 2016. 


\section{RESULTADOS E DISCUSSÕES}

Nos anos de 2017 a 2019 foram notificados 14.546 casos de TB no Pará, com 4.542 casos em 2017, 4.709 em 2018 e 5.295 em 2019. Na PSR foram notificados 190 casos nos três anos, sendo 60 casos em 2017, 70 em 2018 e 60 em 2019.

Os resultados da pesquisa demostram que em ambas as populações os maiores percentuais de notificação foram de casos novos, com a forma da doença pulmonar. Além disso na PSR, o sexo masculino, de cor e/ou raça parda/negra, entre 35 e 44 anos, baixa escolaridade, infectados pelo vírus HIV e que usam drogas além de serem acometidos pela TB, são os que mais abandonam, menos aderem o tratamento e consequentemente morrem da doença.

O perfil sociodemográfico dos casos de TB na PSR analisados nesse estudo se assemelha ao perfil nacional o qual indica a predominância da doença em adultos do sexo masculino, pardos/ pretos e com baixa escolaridade 4 Ademais, estudos indicam que o sexo masculino é o que mais faz uso de substâncias psicoativas e o que menos procura serviços de saúde, isso associado a baixa escolaridade resulta na demora do diagnóstico, a não aceitação da doença e a baixa adesão ao tratamento (ANDRADE et al., 2016).

Os dados obtidos na pesquisa demostram que a PSR em comparação com a PG possui maiores percentuais de reingresso da doença após abandono o que coincide com as taxas de abandono nesta população vulnerável. Estudos potencializam a associação dos casos de retratamento - reingresso após abandono e recidiva com doença ativa - com o surgimento da doença em forma multirresistente (TB MDR) (MICHELETTI et al., 2014)

Os casos de coinfecção TB/HIV deste estudo, chegaram à média de 26,1\% nos três anos pesquisados. Este elevado percentual é resultante da vulnerabilidade social da rua, dieta inadequada, higiene deficiente, condição financeira desfavorável, uso de drogas e vulnerabilidade das pessoas vivendo com HIV. Além disso, os resultados quanto ao uso de drogas ilícitas na PSR, ratificam que a dependência química é uma realidade da rua. O uso de substâncias psicoativas pela PSR representa um desafio para consolidação de políticas públicas e o tratamento de doenças, como HIV, tuberculose e hepatite (HALPERN et al., 2017).

Destaca-se ainda que a PSR obteve menos cura em relação a PG e foi a que mais abandonou o tratamento. O etilismo e o uso de drogas ilícitas em consonância a situação econômica desfavorável são considerados fatores majoritários para abandono do tratamento e, consequentemente não obtenção da cura.

\section{CONCLUSÃO}

A análise do perfil sociodemográfico e clínico-epidemiológico PSR e da PG permitiu observar o quanto a PSR está mais exposta às vulnerabilidades sociais e às consequências da TB. A descontinuidade do tratamento, baixa taxa de cura, maiores taxas de coinfecção TB/HIV e maior 
percentual de morte por TB são fatores que prevaleceram nas PSR.

Por se tratar de uma pesquisa baseada em fontes secundárias, foram encontradas limitações relacionadas aos registros dos casos de $\mathrm{TB}$, com dados importantes para a pesquisa sendo ignorados ou deixados em branco, prejudicando, assim, a realização de uma análise mais consistente e condizente com a realidade. Isso intensifica a invisibilidade social e prejudica o planejamento e implantação de políticas públicas voltada a PSR.

\section{PRINCIPAIS REFERÊNCIAS}

ANDRADE, Aline Teles de et al. Aspectos sociodemográficos de los usuarios de crack asistidos por la red de atención psicossocial. SMAD. Revista eletrônica saúde mental álcool e drogas, v. 12, n. 1, p. 40-47, 2016. Disponível em: http://pepsic.bvsalud.org/scielo.php?pid=S180669762016000100006\&script=sci_abstract\&tlng=es. Acesso em: 12 de Mai. 2020.

BRASIL. Ministério da Saúde. População em situação de rua no Brasil: o que os dados revelam?. 2019. Disponível em: <https://aplicacoes.mds.gov.br/sagirmps/ferramentas/docs/Monitoramento SAGI_Populacao_situacao_rua.pdf $>$.Acesso em: 12 Mai. 2020.

HALPERN, Silvia Chwartzmann et al. Vulnerabilidades clínicas e sociais em usuários de crack de acordo com a situação de moradia: um estudo multicêntrico de seis capitais brasileiras. Cadernos de saúde Pública, v. 33, p. e00037517, 2017. Disponível em: https://www.scielosp.org/article/csp/2017. v33n6/e00037517/. Acesso em: 12 de Mai. 2020.

MICHELETTI, Vania Celina Dezoti et al. Tuberculose resistente em pacientes incluídos no II Inquérito Nacional de Resistência aos Fármacos Antituberculose realizado em Porto Alegre, Brasil. Jornal Brasileiro de Pneumologia, v. 40, n. 2, p. 155-163, 2014. Disponível em: https://www.scielo.br/ scielo.php?pid=S1806-37132014000200155\&script=sci_arttext\&tlng=pt. Acesso em: 12 de Mai. 2020 . 